\title{
Discriminant function analysis of factors related to myocardial infarction in male patients on antihypertensive therapy
}

\author{
A. H. Smith ${ }^{1}$ and F. O. Simpson \\ From the Department of Preventive and Social Medicine and Wellcome Medical Research Institute, De- \\ partment of Medicine, University of Otago Medical School, Dunedin, New Zealand
}

Records of 367 male patients who began attending the Dunedin Hospital Hypertension Clinic between 1959 and 1969 were coded up to the end of 1972. Of these patients, 60 had their first myocardial infarction, or sudden cardiac death without previous evidence of myocardial infarction, while they were attending the clinic. This infarct group was compared with a control group of 120 which was chosen from the remaining patients so that the two groups would be comparable with regard to their age and year of first attendance. $A$ stepwise discriminant function analysis showed that the basal systolic and diastolic pressures measured when patients started attending the clinic were the variables with the most significant difference. Further analysis showed that inclusion of the average casual standing systolic pressure when patients were receiving antihypertensive therapy improved the discrimination between the groups. The average casual standing diastolic pressure during antihypertensive therapy also improved the discrimination, but, curiously enough, with a negative sign in the discriminant function. Quetelet's index of obesity similarly improved the discrimination, obese subjects having less risk of infarction or sudden death. Serum cholesterol, however, was not related to prognosis.

Prospective studies have shown that individuals with hypertension have a higher risk of developing cardiovascular disease than individuals with lower levels of blood pressure (Kannel, 1975). There is also evidence that the treatment of hypertension reduces the incidence of some forms of cardiovascular disease including strokes and congestive heart failure (Veterans Administration Cooperative Study Group, 1967, 1970, 1972). However, doubt remains about the effect of treatment for hypertension on the incidence of myocardial infarction and sudden coronary deaths. In view of this, it was decided to study the records of patients being treated for hypertension and who experienced myocardial infarctions or sudden cardiac deaths. A comparison could then be made with control patients who were also receiving treatment for hypertension, but who had not had myocardial infarctions or sudden cardiac deaths before the end of the study period.

Received 5 April 1976.

${ }^{1}$ Present address: Department of Epidemiology, School of Public Health, The University of North Carolina at Chapel Hill, North Carolina 27514, U.S.A.

\section{Patients and methods}

The patients studied were the 493 male patients who started attending the Dunedin Hospital Hypertension Clinic in the years 1959 to 1969. A total of 126 of these patients were not included in the analysis because they were over 70 when first seen, or were assessed but not treated, or had some other disease not related to hypertension when they first attended. The records of the remaining 367 patients were coded for computer analysis. Among the data items coded, the following have been used in the present analysis.

\section{Patient identification}

Initial investigations Weight, height; casual lying systolic and diastolic blood pressure; basal lying systolic and diastolic blood pressure.

Continuing investigations Cholesterol, urea; casual standing systolic and diastolic blood pressure (either a single reading or the first reading at a halfday test). If there were more than one half-day test 
recorded in any month, the first one was used. If there were no half-day tests in a given month then the first single casual pressure recorded in the month was taken.

Clinical events Myocardial infarction, established stroke, cause of death.

This coding was done retrospectively in 1973, but the format of the clinical records of patients attending this clinic had ensured that a high proportion of the relevant data was available.

Among the 367 patients studied, there were 60 who either had their first myocardial infarction (determined by standard electrocardiographic criteria) while attending the clinic, or who had sudden deaths (usually, but not invariably, with antecedent chest pain) without previous evidence of myocardial infarction. In 7 cases there was some doubt with regard to their inclusion. Four of these had possibly had earlier myocardial infarctions. In the other 3 cases, evidence for a cardiac cause of death was not definite. This doubtful group of 7 did not differ significantly from the remaining 53 as far as the variables used in the analysis were concerned, and they were, therefore, included. However, no patient who had a major stroke before his first myocardial infarction or sudden death, and no patient who had attended for less than 12 months before his first myocardial infarction or sudden death, was included. Of the 60 patients in the group, 33 died suddenly or within one month of the infarct. Most deaths occurred out of hospital and there were only 9 necropsies in these 33 patients.

A control group was selected from the remaining patients in a manner that would avoid confounding the effects of age and changes in patient management, which may have occurred over the study period.

The following arbitrary control-selecting function was used:

matchscore $=3 \times$ absolute difference in year of first attendance + absolute difference in year of birth.

A control was selected for each infarct patient by random selection from those non-infarct patients with whom he had a minimum matchscore, excluding any patient who had a major stroke. The process was then repeated once, so as to give a control group of 120 patients. The mean age of the infarct patients at their first attendance was 58.0 years, and that for the controls 56.8 years. The average date of first attendance for both groups was in the first half of 1963. The infarct group and the control group are thus comparable as far as age and year of first attendance at the Hypertension Clinic are concerned.
The variables used in analysis are defined as follows:

$A S Y S$ : For an infarct patient, this is the average casual standing systolic blood pressure for the 12 months before the month in which he had a myocardial infarction or sudden cardiac death. For a control group patient, it is a similar average of twelve months' casual standing systolic recordings. The period chosen corresponds to the same duration of attendance as that from which ASYS was obtained for the infarct patient with whom he was matched.

$A D I A$ : The average casual standing diastolic pressure corresponding to ASYS.

$B S Y S$ : The basal systolic blood pressure which is measured in the following manner. The patient is admitted to a hospital single room at about 8 p.m. At 10 p.m. he is given $0.2 \mathrm{~g}$ pentobarbitone by mouth; if the patient is awake during the night, a further dose of $0.1 \mathrm{~g}$ pentobarbitone is given, but not later than 4.30 a.m. A technician enters at 7.30 to $8.00 \mathrm{a.m}$. and, without conversing, records the patient's blood pressure every minute for 15 to 20 minutes. The lowest systolic pressure recorded is selected as BSYS.

$B D I A$ : The diastolic pressure corresponding to BSYS. Most patients had either not had previous treatment for hypertension, or they had their treatment stopped before the basal recordings were taken. However, 3 of the 60 infarct patients, and 8 of the 120 control patients were on some antihypertensive treatment at the time of their basal recordings.

CSYS: The casual lying systolic blood pressure recorded during a patient's first attendance at the clinic.

$C D I A$ : The diastolic pressure which corresponds to CSYS.

CHOL: The average serum cholesterol recorded up to the month of myocardial infarction or sudden death. For a control patient, it is the average cholesterol in the period corresponding to that from which CHOL was obtained for the patient used to select him. Cholesterols were not routinely obtained until 1963 and, particularly in the first few years it was measured, some patients may not have been fasting when blood samples were obtained. However, because of the fact that the infarct and control group are comparable as far as year of first attendance is concerned, it is unlikely that any bias has been introduced.

UREA: The serum urea value obtained when a patient first attended the clinic. 
QUET : Quetelet's index, obtained from a patient's weight in grams when he first attended, divided by the square of his height in centimetres.

The method of analysis used was stepwise discriminant analysis, a good account of which is given by Afifi and Azen (1972). The objective of the method in the present context is to select variables which show the most significant difference between the infarction and control groups. At each step, the variable selected is the one that gives the greatest improvement in discrimination, given those variables already selected. The computer programme used for this analysis belongs to the BMD package (Dixon, 1967).

Missing values were dealt with by the method of mean substitution (Chan and Dunn, 1972). A patient in the infarct group who had a missing value for a variable was assigned the mean of that variable for infarct patients, and missing values in the control group were assigned means for the control group. The discriminant analysis was also done a second time with missing values being assigned the overall means for the two groups combined. The results did not affect the conclusions made and so the results of the first analysis only will be presented.

\section{Results}

The means of the variables for the 60 patients in the infarct group and the 120 patients in the control group are shown in Table 1, together with the number of missing values for each variable. The $F$ values obtained during the first step of the discriminant function analysis programme are shown in Table 2. The largest $F$ value is that for the basal diastolic blood pressure, which, therefore, gets selected first into the discriminant function. It should be noted that the $F$ value for the basal systolic blood pressure is almost as large; the fact

TABLE 1 Mean values for infarct group and control group, together with number of missing values of each variable

\begin{tabular}{lccc}
\hline Variable & $\begin{array}{l}\text { Means in } \\
\text { infarct group }\end{array}$ & $\begin{array}{l}\text { Means in } \\
\text { control group }\end{array}$ & $\begin{array}{l}\text { No. of } \\
\text { missing values }\end{array}$ \\
\hline BSYS & 160.8 & 146.6 & 5 \\
BDIA & $102 \cdot 8$ & 93.7 & 5 \\
CHOL & 243.7 & 238.8 & 35 \\
UREA & 40.9 & 39.3 & 0 \\
QUET & 2.61 & 2.68 & 36 \\
CSYS & 206.6 & 196.2 & 3 \\
CDIA & 121.1 & 116.0 & 3 \\
ASYS & 157.3 & 149.9 & 6 \\
ADIA & 97.1 & 97.6 & 6 \\
\hline
\end{tabular}

TABLE $2 \quad F$ values obtained in first step of stepwise discriminant function analysis and statistical significance

\begin{tabular}{lcl}
\hline Variable & $F$ values & $\begin{array}{l}\text { Statistical } \\
\text { significance } P<\end{array}$ \\
\hline BDIA & 14.9 & 0.01 \\
BSYS & 13.4 & 0.01 \\
ASYS & 7.9 & 0.01 \\
CSYS & 5.6 & 0.05 \\
CDIA & 3.6 & \\
QUET & 2.1 & \\
CHOL & 0.7 & \\
UREA & 0.3 & \\
ADIA & 0.1 & \\
\hline
\end{tabular}

that the basal diastolic gets selected first does not mean that it is a significantly better discriminator than the basal systolic. Among the other entries in Table 2, it can be seen that the differences between the groups in the means of the variables ASYS and CSYS are also statistically significant. The remaining variables do not show significant differences when they are considered independently. In particular, it can be seen that ADIA and CHOL fall into this category.

The $F$ values obtained in the fourth step of the analysis are shown in Table 3 . Four variables have been entered into the discriminant function in the order in which they are presented in this table: BDIA, ASYS, ADIA and QUET. The analysis must stop here as none of the $F$ values for the variables not entered approaches statistically significant levels. In contrast, the $F$ values for the variables entered are all significant beyond the 0.01 probability level.

In a separate analysis, the linear discriminant

TABLE $3 F$ values obtained in fourth step of discriminant function analysis for variables entered into discriminant function, and for those not entered

\begin{tabular}{lll}
\hline Variable entered & $F$ values & $\begin{array}{l}\text { Statistical } \\
\text { significance } P<\end{array}$ \\
\hline BDIA & 19.3 & 0.01 \\
ASYS & 14.0 & 0.01 \\
ADIA & 8.9 & 0.01 \\
QUET & 7.3 & 0.01 \\
Not entered & & \\
CHOL & 1.1 & \\
UREA & 1.1 & \\
BSYS & 0.8 & \\
CDIA & 0.6 & \\
CSYS & 0.1 & \\
\end{tabular}


function involving the variables ASYS and ADIA was found to be 2.2 ASYS to 2.3 ADIA. In other words, the best linear discriminator involving these two variables alone is the pulse pressure. It has already been found (Table 1) that ADIA has approximately the same mean in the two groups. It was not until ASYS had been entered into the discriminant function in the stepwise procedure that it became apparent that ADIA would also be included.

Finally, the relation of the variables with the severity of infarction was investigated by considering those patients who had sudden deaths, or who died within one month of their myocardial infarction. Altogether 33 patients fall into this category and their means are compared with the means for the controls selected along with them in Table 4. A similar comparison involving the remaining 27 infarct patients is also presented in this table. It can be seen that many of the differences between infarct and control groups are more pronounced in the infarct patients who died within one month. Stepwise discriminant analysis for this group of 33 with their 66 controls followed a pattern similar to that presented for the whole group. In contrast, the stepwise analysis for those who did not die within one month stopped after basal diastolic was placed in the discriminant function. Numbers are inadequate to warrant presenting separate discriminant analysis results, but it is apparent that the differences between the infarct group and the control group can be largely attributed to patients with more severe disease who died within one month of their first myocardial infarction, or who had sudden cardiac deaths without previous myocardial infarction.

TABLE 4 Mean values for infarct patients who died within one month, and for those who survived more than one month.

\begin{tabular}{|c|c|c|}
\hline Variable & $\begin{array}{l}\text { Patients (33) who } \\
\text { died within one } \\
\text { month }\end{array}$ & $\begin{array}{l}\text { Patients (27) who } \\
\text { survived at least } \\
\text { one month }\end{array}$ \\
\hline $\begin{array}{l}\text { BSYS } \\
\text { BDIA } \\
\text { CHOL } \\
\text { UREA } \\
\text { QUET } \\
\text { CSYS } \\
\text { CDIA } \\
\text { ASYS } \\
\text { ADIA }\end{array}$ & $\begin{array}{r}168.1(147.7) \\
103.4(93.7) \\
247.2(234.7) \\
44.1(39.1) \\
2.58(2.69) \\
210.9(195.4) \\
117.9(113.9) \\
160.8(150.5) \\
95.1(97.0)\end{array}$ & $\begin{array}{r}151.9(145.3) \\
102.0(93.7) \\
237.9(244.8) \\
37.0(39.6) \\
2.64(2.66) \\
201.4(197.2) \\
125.0(118.4) \\
153.3(149.1) \\
99.4(98.3)\end{array}$ \\
\hline
\end{tabular}

Note \&ariable means for relevant control groups are shown in parentheses.

\section{Discussion}

Considerable speculation has centred on the effect of antihypertensive treatment on the incidence of coronary heart disease. The known effect of treatment on the incidence of other forms of cardiovascular disease makes it unethical to conduct controlled trials for anything but very mild hypertension. Such studies also require large numbers of patients (Peart, 1973) and negative findings will not prove that the treatment of more severe hypertension does not reduce the incidence of coronary heart disease. It is felt that the results we have presented indicate that valuable information in this regard may be obtained by studying patients already receiving treatment for their hypertension.

The study of prognosis of hypertension is complicated by the possibility of confounding the effects of age with the effects of other variables. Kleinbaum et al. (1971) attempted to solve this problem by including second order product terms in their stepwise discriminant analysis and found that diastolic blood pressure, age, and cholesterol $x$ age, were chosen for their discriminant function. Unfortunately, product terms of this nature pose difficulties in interpreting the results of analysis. We have avoided this problem by selecting a control group which is comparable with the infarct group as far as age and year of first attendance at the clinic are concerned.

The measurement of the basal blood pressure (Alam and Smirk, 1943) when patients first attend has been the practice at the Dunedin Hospital Hypertension Clinic for many years (Smirk, 1957; Smirk, Veale, and Alstad, 1959; Hodge and Smirk, 1967). The present results once again confirm its usefulness as a measure of the severity of hypertension. In particular, it is a considerably better indicator of the risk of coronary heart disease than the first casual recording.

The relation between the risk of infarction and blood pressure levels on treatment is of greater importance. The results we obtained indicate that the average standing systolic blood pressure on treatment is related to the incidence of myocardial infarction and sudden cardiac death. On the other hand, the average standing diastolic blood pressure does not discriminate between the groups when it is considered on its own. Beevers, Fairman, and Harpur (1973) also found that the control of hypertension as measured by the average diastolic blood pressure was not related to the incidence of myocardial infarction. Breckenridge, Dollery, and Parry (1970) considered the average systolic and diastolic blood pressures on treatment and showed that there were no consistent differences between 
patients dying from uraemia, cerebrovascular disease, or myocardial infarction. However, they found that problems of handling large amounts of data prevented comparison between these patients and the rest of their clinic patients.

In spite of the fact that the average diastolic pressure does not discriminate between our two groups, we found that the average pulse pressure (i.e. the difference between the average systolic and diastolic pressures) improves the discrimination achieved by the average systolic alone. It is of interest to note that Kannel, Gordon, and Schwartz (1971b) found that the diastolic blood pressure generally had a negative sign in their discriminant functions. However, in their untreated population, the pulse pressure did not give a better discrimination than the systolic pressure. Our own findings relating to pulse pressure must be interpreted with some caution, because its mean difference between the groups is not much greater than that for the systolic pressure alone (Table 1). Before attaching too much significance to it, the sort of analysis we have done must be replicated in studies involving other patients.

It is of particular interest that the average blood pressures when patients are receiving antihypertensive therapy are included in the discriminant function, even though a basal pressure has already been selected. This suggests that, even allowing for differences before treatment, the degree of control of hypertension by therapy affects the incidence of myocardial infarction and sudden coronary death. A separate analysis (Smith, 1975) showed that patients with an average systolic pressure below $170 \mathrm{mmHg}$ all had approximately the same risk of infarction, but a patient with an average systolic pressure above this level was exposed to a greater risk. An even greater risk differential was found when comparing those with an average pulse pressure above $70 \mathrm{mmHg}$ with those whose average pulse pressure was below $70 \mathrm{mmHg}$. These levels were calculated using all 367 patients in this study with all ages pooled, and a larger number of cases is required to determine their relation to age.

We were also interested to find that Quetelet's index gets included in the discriminant function by the stepwise analysis. Its very low correlation with height in our patients of -0.08 is close to that found in other studies (Goldbourt and Medalie, 1974). It appears in the discriminant function with a negative sign after the basal and average treatment levels have been included. The implication is that, for given pre-treatment and treatment blood pressure levels, the more obese the patient the less likely he is to have a coronary event. This result must be interpreted with some caution as there are a considerable number of missing values for this variable (Table 1). Account must also be taken of the fact that obesity commonly has a negative correlation with smoking (Keys et al., 1972). We did not have adequate data to include smoking in our analysis and it may be that the apparent association of prognosis with obesity is in fact the result of the effects of smoking. However, there are some previous reports that suggest that in hypertension, particularly in women patients, obesity is associated, with a better prognosis (Simpson and Gilchrist, 1958; Nye, 1964).

The fact that serum cholesterol is not included in the discriminant function warrants some comment. Kannel et al. (1971a) found that raised serum cholesterol level is associated with an increased incidence of coronary heart disease in the Framingham study but this association occurred predominantly in the younger age-groups. In the 50 to 62 year age-group, on the other hand, the difference in the mean cholesterol between their coronary heart disease group and their non-coronary heart disease group was only $0.39 \mathrm{mmol} / 1(15.4 \mathrm{mg} / 100$ $\mathrm{ml}$ ) (Kannel et al., 1971a). A study of the regression coefficients they obtained also indicates that, though the cholesterol levels were related to the incidence of cardiovascular disease, their relation to cardiovascular mortality was not significant (Kannel and Dawber, 1974). Our own results indicate that it is not a useful predictor of the incidence of myocardial infarction and sudden cardiac deaths in male patients on antihypertensive therapy.

Another variable which has been used as a prognostic indicator in hypertension is retinal grade. This variable was included in our first stepwise discriminant analysis, but at no stage did it look likely to be selected for the discriminant function. The fact that it is a discrete variable means that it should not be included in a stepwise discriminant function analysis, unless the sole purpose is to obtain a predictive formula. Because of this, a separate analysis was done using a stepwise information theory analysis (A. H. Smith and P. V. de Souza, unpublished observations). This also gave the impression that blood pressure measurements are considerably better prognostic indicators than the retinal grade.

Finally, it was shown that the differences in blood pressure levels between the infarct patients and the controls were greatest for those who had sudden coronary deaths or who died within one month of their first myocardial infarction. In fact, the discrimination achieved between those who had a myocardial infarct and survived more than one month and their control group is not good and involves the basal diastolic pressure alone. The 
numbers studied are small, but it appears that one of the effects of adequate control of blood pressure is to reduce the incidence of sudden coronary deaths and myocardial infarctions which are fatal within one month.

We thank Professor C. W. Dixon, Mr. G. F. S. Spears, Dr. D. C. E. Manley and Mr. P. V. de Souza for helpful comments and suggestions at various stages of this study. Many physicians have contributed to the Hypertension Clinic records, especially Sir Horace Smirk, Dr. J. V. Hodge, Dr. Hendrika J. Waal-Manning, Dr. J. K. McKenzie and Dr. J. $\mathrm{Ng}$. We are also grateful for financial support from the Medical Research Council of New Zealand.

\section{References}

Afifi, A. A., and Azen, S. P. (1972). Statistical Analysis. Academic Press, New York.

Alam, G. M., and Smirk, F. H. (1943). Casual and basal blood pressures. I. In British and Egyptian men. British Heart fournal, 5, 152.

Beevers, D. G., Fairman, M. J., and Harpur, J. E. (1973). Antihypertensive treatment and the course of established cerebral vascular disease. Lancet, 1, 1407.

Breckenridge, A., Dollery, C. T., and Parry, E. H. O. (1970), Prognosis of treated hypertension. Quarterly fournal of Medicine, 39, 411.

Chan, L. S., and Dunn, O. J. (1972). Treatment of missing values in discriminant analysis. I. The sampling experiment. Fournal of the American Statistical Association, 67, 473.

Dixon, W. J. (1967). (Ed.) BMD Biomedical Computer Programs, 2nd ed. University of California Press, Berkeley.

Goldbourt, U., and Medalie, J. H. (1974). Weight-height indices. British fournal of Preventive and Social Medicine, 28, 116.

Hodge, J. V., and Smirk, F. H. (1967). The effect of drug treatment of hypertension on the distribution of deaths from various causes. American Heart fournal, 73, 441.

Kannel, W. B. (1975). Role of blood pressure in cardiovascular disease: the Framingham study. Angiology, 26, 1.

Kannel, W. B., and Dawber, T. R. (1974). Hypertension as an ingredient of a cardiovascular risk profile. British fournal of Hospital Medicine, 11, 508.

Kannel, W. B., Garcia, M. J., McNamara, P. M., and Pearson, C. (1971a). Serum lipid precursors of coronary heart disease. Human Pathology, 2, 129.

Kannel, W. B., Gordon, T., and Schwartz, M. J. (1971b). Systolic versus diastolic blood pressure and risk of coronary heart disease. American fournal of Cardiology, 27, 335.
Keys, A., Aravanis, C., Blackburn, H., Van Buchem, F. S. P., Buzina, R., Djordjevic, B. S., Fidanza, F., Karvonen, M. J., Menotti, A., Puddu, V., and Taylor, H. L. (1972). Coronary heart disease: overweight and obesity as risk factors. Annals of Internal Medicine, 77, 15.

Kleinbaum, D. G., Kupper, L. L., Cassel, J. C., and Tyroler, H. A. (1971). Multivariate analysis of risk of coronary heart disease in Evans County, Georgia. Archives of Internal Medicine, 128, 943.

Nye, E. R. (1964). Coronary artery disease in hypertensive patients. New Zealand Medical fournal, 63, 483.

Peart, W. S. (1973). The organization of a multi-centre randomized control therapeutic trial for mild to moderate hypertension. Clinical Science and Molecular Medicine, 45, 67s.

Simpson, F. O., and Gilchrist, A. R. (1958). Prognosis in untreated hypertensive vascular disease. Scottish Medical fournal, 3, 1.

Smirk, F. H. (1957). High Arterial Pressure. Blackwell, Oxford.

Smirk, F. H., Veale, A. M. O., and Alstad, K. (1959). Basal and supplemental blood pressures in relationship to life expectancy and hypertension symptomatology. New Zealand Medical fournal, 58, 711.

Smith, A. H. (1975). A stochastic model and the risk of myocardial infarction in treated hypertension. In J. S. Dodge and S. R. West (Eds.): Epidemiology and Primary Medical Care. Proceedings of the Australian Society for Epidemiological Research in Community Health. Dunedin, May 1975, pp 185-189. Department of Preventive and Social Medicine, University of Otago Medical School, Dunedin, New Zealand.

Veterans Administration Cooperative Study Group on Antihypertensive Agents (1967). Effects of treatment on morbidity in hypertension: results in patients with diastolic blood pressures averaging 115 through 129 mmHg. Fournal of the American Medical Association, 202, 1028 .

Veterans Administration Cooperative Study Group on Antihypertensive Agents (1970). Effects of treatment on morbidity in hypertension. Part 2. Results in patients with diastolic blood pressure averaging 90 through $114 \mathrm{mmHg}$. fournal of the American Medical Association, 213, 1143.

Veterans Administration Cooperative Study Group on Antihypertensive Agents (1972). Effects of treatment on morbidity in hypertension: III. Influence of age, diastolic pressure, and prior cardiovascular disease; further analysis of side effects. Circulation, 45, 991.

Requests for reprints to Professor F. O. Simpson, Department of Medicine, Wellcome Medical Research Institute, Medical School, University of Otago, P.O. Box 913, Dunedin, New Zealand. 